Article

\title{
Genetic Improvement of Torulaspora delbrueckii for Wine Fermentation: Eliminating Recessive Growth-Retarding Alleles and Obtaining New Mutants Resistant to $\mathrm{SO}_{2}$, Ethanol, and High $\mathrm{CO}_{2}$ Pressure
}

\author{
Rocío Velázquez ${ }^{1}{ }^{1}$, Alberto Martínez ${ }^{1}$, Emiliano Zamora ${ }^{2}$, María L. Álvarez ${ }^{2}$, \\ Joaquín Bautista-Gallego ${ }^{1}{ }^{\mathbb{D}}$, Luis M. Hernández ${ }^{1}$ and Manuel Ramírez ${ }^{1, * \mathbb{C}}$ \\ 1 Departamento de Ciencias Biomédicas (Área de Microbiología), Facultad de Ciencias, \\ Universidad de Extremadura, 06006 Badajoz, Spain; rociovelazquez1981@gmail.com (R.V.); \\ amartinetp@alumnos.unex.es (A.M.); joaquin.bautistagallego@gmail.com (J.B.-G.); \\ lmhernan@unex.es (L.M.H.) \\ 2 Estación Enológica, Junta de Extremadura, 06200 Almendralejo, Spain; emiliano.zamora@juntaex.es (E.Z.); \\ luz.alvarez@juntaex.es (M.L.Á.) \\ * Correspondence: mramirez@unex.es; Tel.: +34-924289426
}

Received: 25 August 2020; Accepted: 5 September 2020; Published: 7 September 2020

check for updates

\begin{abstract}
The use of Torulaspora delbrueckii has been repeatedly proposed to improve a wine's organoleptic quality. This yeast has lower efficiency in completing wine fermentation than Saccharomyces cerevisiae since it has less fermentation capability and greater sensitivity to $\mathrm{SO}_{2}$, ethanol, and $\mathrm{CO}_{2}$ pressure. Therefore, the completion of fermentation is not guaranteed when must or wine is single-inoculated with $T$. delbrueckii. To solve this problem, new strains of T. delbrueckii with enhanced resistance to winemaking conditions were obtained. A genetic study of four wine $T$. delbrueckii strains was carried out. Spore clones free of possible recessive growth-retarding alleles were obtained from these yeasts. These spore clones were used to successively isolate mutants resistant to $\mathrm{SO}_{2}$, then those resistant to ethanol, and finally those resistant to high $\mathrm{CO}_{2}$ pressure. Most of these mutants showed better capability for base wine fermentation than the parental strain, and some of them approached the fermentation capability of $S$. cerevisiae. The genetic stability of the new mutants was good enough to be used in industrial-level production in commercial wineries. Moreover, their ability to ferment sparkling wine could be further improved by the continuous addition of oxygen in the culture adaptation stage prior to base wine inoculation.
\end{abstract}

Keywords: Torulaspora delbrueckii; wine fermentation; spore clone; sparkling wine; ethanol resistance; $\mathrm{SO}_{2}$ resistance; pressure resistance

\section{Introduction}

Among non-Saccharomyces yeasts, Torulaspora delbrueckii is probably the one with a wine-fermentation performance closest to Saccharomyces cerevisiae, and therefore the most suitable for winemaking. This yeast has recently been recommended for must fermentation mainly because it improves some wine parameters, such as decreased acetic acid and ethanol production, increased amount of glycerol, increased mannoprotein and polysaccharide release, promotion of malolactic fermentation, increased amounts of wanted aromatic compounds (fruity esters, lactones, thiols, and terpenes), and decreased amounts of unwanted aromatic compounds (such as higher alcohols). The features of this yeast may improve wine quality or complexity (reviewed in [1,2]). In addition, 
it may stimulate malolactic fermentation [3]. However, T. delbrueckii has some serious drawbacks that may discourage its use in winemaking. It has higher rates of $\mathrm{CO}_{2}$ production and $\mathrm{O}_{2}$ consumption than $S$. cerevisiae, which result in low biomass yield from batch cultures and is a handicap for the commercial production of T. delbrueckii [4]. Although both yeasts have very similar patterns for sugar utilization under fully respiratory growth conditions, T. delbrueckii grows more slowly than $S$. cerevisiae under strict anaerobic conditions [5,6]. As a consequence, T. delbrueckii has less fermentation vigor than $S$. cerevisiae under usual wine fermentation conditions, and has serious difficulties in dominating wine fermentation even when initially inoculated at a high proportion (above $10^{7} \mathrm{CFU} / \mathrm{mL}$ ) $[2,4,7]$. These drawbacks are especially serious when making white and sparkling wines, which is usually done under strict anaerobic conditions [2]. Besides this, T. delbrueckii is also less resistant to other stressing conditions closely related to winemaking than $S$. cerevisiae, such as the rapid increase of ethanol concentration, the presence of $\mathrm{SO}_{2}$, and high $\mathrm{CO}_{2}$ pressure. These circumstances negatively affect the fermentation efficiency of $T$. delbrueckii during still or sparkling wine making. As this yeast has poor resistance to the fast increase of ethanol concentration, its fermentation rate slows down and cell death increases once the tumultuous fermentation stage of sugar-rich substrates has been reached [8]. As a consequence, fermentation may slow to become sluggish or even stop, or it may continue because of the participation of contaminating wild Saccharomyces yeasts [7,9]. Such a sequence of events reduces the participation of T. delbrueckii during must fermentation, making the real influence of this yeast on wine composition and quality uncertain [1]. The difference in ethanol resistance between T. delbrueckii and S. cerevisiae is easily visible on Yeast extract-Peptone-Dextrose growth medium (YEPD) plates supplemented with different ethanol concentrations [2]. Nevertheless, some strains of T. delbrueckii can dominate and complete crushed grape fermentation under specific favorable conditions (low amounts of competitor S. cerevisiae yeasts, large inoculum of healthy T. delbrueckii cells, frequent shaking to provide extra oxygen, addition of extra amounts of nutrients, and low amount of $\mathrm{SO}_{2}$ ) to reach greater than $14 \%$ ethanol concentration [2,10]. Even under these favorable conditions, T. delbrueckii fermentation takes much longer to complete than S. cerevisiae fermentation. However, as the rate of rise of ethanol production is rather low, part of the T. delbrueckii population has a chance to progressively become adapted to the stressing conditions and be able to complete grape fermentation.

As for ethanol, $\mathrm{SO}_{2}$ is another important stress factor since this compound is used during winemaking as a microbial inhibitor and antioxidant. Therefore, $\mathrm{SO}_{2}$ resistance is a desired trait for wine yeast strains [11]. The lower $\mathrm{SO}_{2}$ resistance of T. delbrueckii with respect to S. cerevisiae is clearly visible on Synthetic Defined medium (SD) agar plates supplemented with different concentrations of this compound. Despite this, T. delbrueckii is able to complete fermentation in the presence of $50 \mathrm{mg} / \mathrm{L}$ $\mathrm{SO}_{2}$. However, a concentration of $125 \mathrm{mg} / \mathrm{L} \mathrm{SO}_{2}$, as is frequently used in winemaking, is lethal for T. delbrueckii [2].

Recently, trials using T. delbrueckii for sparkling wine making have been carried out. Some strains of this yeast can be inoculated in the first-fermentation of grape must to obtain base wine with improved quality $[7,12]$. However, these strains were unable to complete the second-fermentation of the base wine because they cannot survive above $3.5 \mathrm{~atm}$ of $\mathrm{CO}_{2}$ pressure inside a glass bottle of sparkling wine [12]. As a consequence, base wine single-inoculated with $T$. delbrueckii only completed second-fermentation when there were contaminant Saccharomyces yeasts, which became dominant after $\mathrm{CO}_{2}$ pressure rose above $3 \mathrm{~atm}$, while T. delbrueckii progressively became inviable [2]. Therefore, high $\mathrm{CO}_{2}$ pressure is another factor that decreases the competitiveness of $T$. delbrueckii compared to its potential competitor S. cerevisiae. This suggests that its resistance to high pressure should be improved if any dominance of T. delbrueckii during second fermentation of sparkling wine is intended.

Based on our experience, the isolation of genetically stable mutants resistant to high ethanol concentrations is not an easy task. It is possible to isolate $S$. cerevisiae clones that are resistant to as much as $19 \%$ ethanol. However, these clones seem to be yeasts that adapt slowly to growth in these stressing conditions, and do not maintain the ethanol resistance phenotype once they are newly grown in the absence of ethanol and put back into media with high ethanol concentrations. Although changes 
of expression in a single gene may generate the tolerant phenotype $[13,14]$, this occurs with difficulty since the tolerance to stressors requires changes of expression for many genes [15]. Besides this, yeast tolerance to high ethanol concentrations is temperature dependent since both factors exert a synergistic negative effect on yeast growth and enzymes [16]. Additionally, other environmental factors such as the nutrients available, osmotic pressure, or the way the carbohydrate substrate is added (sequentially, or all at the time of yeast inoculation) may also dramatically influence yeast ethanol tolerance [17-19]. Increasing the $\mathrm{SO}_{2}$ tolerance in yeast may also interfere with basic cellular metabolism and involve an interplay of genetic changes. Although some yeasts, including S. cerevisiae, have developed physiological mechanisms for $\mathrm{SO}_{2}$ tolerance, some of these molecular mechanisms are also complex and have only recently been investigated. Among them are sulfite reduction, sulfite oxidation, acetaldehyde production, sulfite efflux, and behaving as viable but not culturable cells [20]. In addition, $\mathrm{SO}_{2}$ tolerance has been connected to a molecular mechanism which involves a higher transcription level of the SSU1 gene [21-23]. The molecular mechanisms involved in $\mathrm{CO}_{2}$ pressure tolerance have only very recently been investigated. Although few details are known as yet, the data available indicate that one is also facing a complex situation involving the stress sub-proteome, cell viability, and metabolites such as glycerol, reducing sugars, and ethanol [24].

Whereas S. cerevisiae's fermentative lifestyle is shared by other fermentative yeasts [25], its ethanol tolerance is only shared with other Saccharomyces yeasts [26]. A similar situation may be the case for $\mathrm{CO}_{2}$ pressure tolerance since Saccharomyces are the only known yeasts able to successfully complete the second fermentation of sparkling wine inside a hermetically closed glass bottle. Contrarily, the $\mathrm{SO}_{2}$ tolerance of S. cerevisiae is shared with other non-Saccharomyces fermentative yeasts. As strains of yeast are phenotypically diverse, the isolation and selection of $T$. delbrueckii strains resistant to any of the aforementioned stresses should be possible. Thereafter, genetic improvement through yeast breeding or the elimination of non-interesting alleles could be carried out. However, the lack of precise knowledge about the lifecycle of this yeast makes it difficult to design strategies for biotechnological improvement by using the classical genetic techniques already used for $S$. cerevisiae wine yeasts $[27,28]$. Besides this, theoretically, one could try to get $T$. delbrueckii to evolve similarly as S. cerevisiae did to improve ethanol, $\mathrm{SO}_{2}$, and $\mathrm{CO}_{2}$ pressure tolerance, as has been done by adaptive laboratory evolution to improve growth and ethanol production at high temperatures [13].

The present work describes a genetic study of four selected T. delbrueckii strains to eliminate possible recessive deleterious alleles in order to obtain new improved spore clones with enhanced fermentation capability. Then, we performed sequential isolations of spontaneous mutants resistant to different stressful conditions related to still and sparkling wine making. The main aim was to improve the overall fermentation performance of this yeast species to bring it as close as possible to that usually shown by S. cerevisiae wine yeasts. The utility of some of these improved mutants for commercial winery applications will be discussed.

\section{Materials and Methods}

\subsection{Yeast Strains}

S. cerevisiae (Sc) EX229 is a Klus-killer wine strain that kills other S. cerevisiae and T. delbrueckii yeasts [29]. Sc 85R4A is a non-killer, cycloheximide-resistant $\left(\mathrm{cyh}^{\mathrm{R}}\right)$ spore clone obtained from the $S c$ EX85R (originally named JP85R; [30]) wine yeast. These S. cerevisiae strains were used in this study as reference yeasts for still and sparkling wine fermentation. T. delbrueckii (Td) Kbarr EX1180 and Td EX1257 are prototrophic wine yeasts that kill all known types of S. cerevisiae killer and non-killer strains and non-killer T. delbrueckii strains. Td EX1180-11C4 and Td EX1257-CYH5 are cyh ${ }^{\mathrm{R}}$ spontaneous mutants from $T d$ EX1180 and $T d$ EX1257, respectively. These strains had previously been selected for winemaking $[9,10,12]$. The genetic marker $\mathrm{cyh}^{\mathrm{R}}$ allows easy traceability of the new mutants obtained from these yeasts. Industrial use of $T d$ EX1180 and $T d$ EX1257 is under patent application [31]. 


\subsection{Culture Media and Phenotype Tests}

Standard culture media were used for yeast growth [32]. YEPD broth contained 1\% yeast extract, $2 \%$ peptone, and $2 \%$ glucose. YEPD agar is YEPD broth with $2 \%$ agar. YEPD+cyh is YEPD agar supplemented with $2 \mu \mathrm{g} / \mathrm{mL}$ cycloheximide [30]. YEPD+EtOH is YEP -agar supplemented with ethanol just before pouring the medium into Petri plates to a $5 \%$ or $10 \%(v / v)$ final concentration. SD agar contained $0.67 \%$ Yeast Nitrogen Base (without amino acids; with ammonium sulfate, Difco), $2 \%$ glucose and $2 \%$ Bacto-agar. $\mathrm{SD}+\mathrm{SO}_{2}$ is $\mathrm{SD}$ agar buffered with $75 \mathrm{mM}$ tartaric acid at $\mathrm{pH} 3.5$, and supplemented with a freshly prepared $6 \% \mathrm{~K}_{2} \mathrm{~S}_{2} \mathrm{O}_{2}$ stock solution that was added to each plate two hours before yeast seeding (125 or $250 \mathrm{mg} / \mathrm{L} \mathrm{SO}_{2}$ final concentration) [28]. Macabeo grape must (21.3 ${ }^{\circ} \mathrm{Brix}, \mathrm{pH} 3.2$, malic acid $1.4 \mathrm{~g} / \mathrm{L}$, lactic acid $0.08 \mathrm{~g} / \mathrm{L}$ ) was sterilized by membrane filtration through a Millipore system (0.45 $\mu \mathrm{m}$ membrane). This sterile synthetic must was a modified version [33] of that described previously [34]. The sterile synthetic base wine used contained $1 \%$ yeast extract, $0.1 \%$ peptone, and $2.4 \%$ sucrose, $10 \%$ ethanol, $0.3 \%$ tartaric acid, and $0.2 \%$ malic acid, $\mathrm{pH} 3.1$.

Standard procedures were used for the sporulation of yeast cultures and dissection of asci [35]. Yeast cells were grown on YEPD agar plates or in YEPD broth for two days at $30{ }^{\circ} \mathrm{C}$ and then transferred to sporulation plates (SPO; $1 \%$ potassium acetate, $0.1 \%$ yeast extract, $0.05 \%$ glucose, $2 \%$ agar) and incubated for 20 days at $25{ }^{\circ} \mathrm{C}$. The percentage of sporulated yeasts was determined at this time. The tetrads (asci) from each yeast strain were dissected on YEPD plates using a micromanipulator, and then incubated for 5 days at $30^{\circ} \mathrm{C}$. Spore viability and spore clone size were determined at this time. Spore clones were tested for phenotype segregations by replica plating on agar plates for sugar fermentation (sucrose, maltose, galactose, melibiose, melezitose, trehalose, raffinose, starch and $\alpha$-methyl glucoside), copper resistance, $\mathrm{SO}_{2}$ resistance, and $\mathrm{H}_{2} \mathrm{~S}$ production as previously described [36]. Homothallism was determined by examining the ability of isolated spore clones to sporulate. Petite phenotype was analyzed on Yeast extract-Peptone-Glycerol medium (YPG) (1\% yeast extract, $2 \%$ peptone, $3 \%$ glycerol, $2 \%$ agar, and $1 \%$ ethanol added after autoclaving).

\subsection{Grape Must, Synthetic Must and Synthetic Base Wine Fermentations}

Yeast cells were cultured in YEPD broth for 2 days at $30^{\circ} \mathrm{C}$, washed twice with sterile water, and inoculated into synthetic must or synthetic base wine $\left(2-4 \times 10^{6}\right.$ cells $/ \mathrm{mL}$ for $S$. cerevisiae, and $2-4 \times 10^{7}$ cells $/ \mathrm{mL}$ for T. delbrueckii). In parallel, a fermentation inoculated with Sc EX229 or Sc 85R4A was done as a reference positive control for each experiment. Fermentations were performed in $250 \mathrm{~mL}$ Erlenmeyer flasks with $60 \mathrm{~mL}$ of must or base wine, at $20^{\circ} \mathrm{C}$ or $16-18{ }^{\circ} \mathrm{C}$, respectively. Before base wine inoculation, when required, yeast cultures were adapted to growth in this medium as indicated below for sparkling wine making, using the different culture shaking procedures mentioned in the Results section. The density, ${ }^{\circ}$ Brix, yeast growth (total and viable yeast cells), and dead cells were monitored. Cell death was determined by staining with methylene blue and the observation of yeast samples under a microscope with a Nomarski $60 \times$ objective. Since the morphological changes in the yeast cells during the second fermentation of sparkling wine was highly variable, the total amount of dead cells was calculated as the sum of blue, empty, and destroyed/autolyzed cells [12].

\subsection{Base Wine Fermentation for Sparkling Wine Making}

Cava-type sparkling wine was made using the traditional method in our experimental winery as previously described [12]. Two different commercial base wines were used, one from Garnacha red grapes ( $\mathrm{pH} 3.20,4.93 \mathrm{~g} / \mathrm{L}$ total acidity, $0.87 \mathrm{~g} / \mathrm{L}$ reducing sugars, $10.9 \%$ alcohol $v / v$ ) and another from Macabeo white grapes ( $\mathrm{pH} 3.18,5.7 \mathrm{~g} / \mathrm{L}$ total acidity, $1.2 \mathrm{~g} / \mathrm{L}$ reducing sugars, $10.8 \%$ alcohol $v / v$ ). Before base wine inoculation, each yeast culture was adapted to growth in each base wine as follows. Each yeast pellet from a 48-h YEPD broth culture was resuspended in sterile water supplemented with $2.4 \%$ sucrose and $0.2 \%$ diammonium phosphate $\left((\mathrm{NH} 4)_{2} \mathrm{PO} 4\right)\left(2-4 \times 10^{9} \mathrm{CFU} / \mathrm{mL}\right)$, and incubated at room temperature $\left(18-22{ }^{\circ} \mathrm{C}\right)$ for about $2 \mathrm{~h}$. This culture was then diluted with one volume of a 
mixture 1:1 water:base wine with $2.4 \%$ sucrose, and incubated for $5 \mathrm{~h}$ at $18-22{ }^{\circ} \mathrm{C}$. Each culture was then diluted again with one volume of base wine with $2.4 \%$ sucrose and incubated overnight at room temperature. Finally, each culture was diluted again with 1.5 volumes of base wine with $2.4 \%$ sucrose, and incubated for $5 \mathrm{~h}$ at $18-22{ }^{\circ} \mathrm{C}$. Occasional shaking of these cultures was done every $2-12 \mathrm{~h}$ as convenient. These yeast-adapted cultures contained a final cell concentration of $2-6 \times 10^{8} \mathrm{CFU} / \mathrm{mL}$. For sparkling wine making, base wine was supplemented with $2.4 \%$ sucrose and $0.02 \%$ diammonium phosphate, and single-inoculated with each adapted yeast culture in $0.75 \mathrm{~L}$ capped bottles in which high pressures above $6 \mathrm{~atm}$ could be reached after the second fermentation. Three replicates of each yeast's second fermentation were done. The intended amount of yeast inoculum was $2-4 \times 10^{6}$ viable cells $/ \mathrm{mL}$ for S. cerevisiae, and $2-4 \times 10^{7}$ viable cells $/ \mathrm{mL}$ for $\mathrm{T}$. delbrueckii. The second fermentation was done at $18-19^{\circ} \mathrm{C}$ for the first 15 days, and thereafter at $12-14^{\circ} \mathrm{C}$. Samples for microbiological and chemical assay were taken at different times from 0 to 270 days. After 270 days of fermentation and aging, the sparkling wines were riddled for 30 days to move the lees to the bottle neck. Finally, after disgorging, aromatic compounds and organoleptic quality were assayed. A descriptive organoleptic analysis was done for each sparkling wine by an expert panel of 10 judges as previously described [9].

\subsection{Determination of the Inoculated Yeast Proportion during Fermentation}

The percentages of $\mathrm{cyh}^{\mathrm{R}}$ genetically marked Sc 85R4A, Td EX1180-11C4, and Td EX1257-CYH5 yeasts were determined by replica-plating on YEPD+cyh [37]. The percentage of wild yeasts such as Sc EX229 was determined by mtDNA restriction pattern analysis [38]. When appropriate, this same procedure was used to validate the results obtained by replica-plating analysis.

\subsection{Analytical Methods}

Degrees Brix ( ${ }^{\circ}$ Brix) were measured using a digital refractometer. Alcohol content, $\mathrm{pH}$, total acidity, volatile acidity, glucose and fructose, and density were determined using European Commission (EC) recommended methods [39]. Sparkling wine pressure was measured at room temperature using an aphrometer, and values were then corrected to $20^{\circ} \mathrm{C}$ by using Henry's law constant.

\section{Results and Discussion}

\subsection{Genetic Characterization of T. delbrueckii: Sporulation Capability, Viability of Spores, Presence of Recessive Growth-Retarding Alleles, and Degree of Heterozygosity}

The sporulation of T. delbrueckii in SPO medium was rather low (3.5-22\% tetrads) compared to that of S. cerevisiae (55-74\%). However, the viability of T. delbrueckii spores after tetrad dissection on YEPD agar was generally high (average 78\%,68\%,64\%, and 69\% for Td EX1180, Td EX1180-11C4, Td EX1257, and Td EX1257-CYH5, respectively), although not as high as that of S. cerevisiae, which most times was 100\%. Most T. delbrueckii spore clones were large in size. Only two spore clones were medium to small in size and sectored, similar to those previously found for genetically unstable yeasts [28,40] (Figure 1A). Despite this, no pattern of genetic segregation for non-viable or small colonies bearing deleterious recessive alleles was found in $T d$ EX1180. The phenotype of these two clones could therefore be due to some spontaneous mutation or metabolic deficiency that happened to affect some cells that then did not multiply or died, causing circular sectors of non-growth in the colony (i.e., no deleterious allele was detected that would be responsible for slow growth in rich medium, nor was a regular pattern for genetic segregation of non-viable spores found). If any deleterious or lethal alleles existed in any of these T. delbrueckii strains, they could be associated with spores that did not germinate. In view of these results, it does not seem reasonable to try to select spore clones free of recessive growth-retarding alleles as a basic, and much less exclusive, strategy to address the genetic improvement of these T. delbrueckii strains, contrary to the case previously described for S. cerevisiae wine strains [28]. 


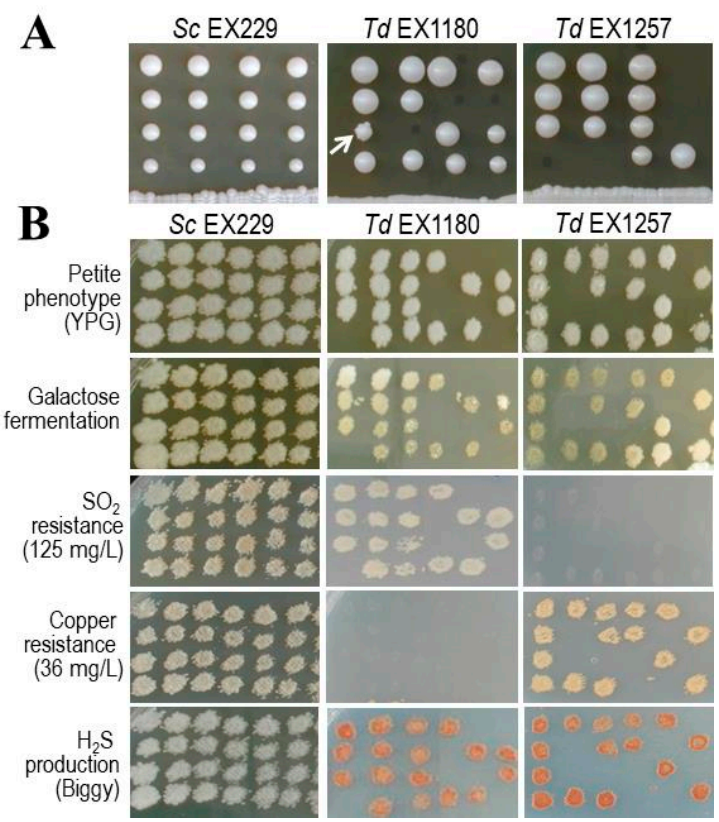

Figure 1. Genetic characterization of T. delbrueckii. (A) Spore colony size after tetrad dissection of Sc EX229, and Td EX1180 and Td EX1257 strains. Spore tetrads from separate asci are arranged along vertical lines. Sectored colony is indicated with an arrow. (B) Examples of some phenotype tests after replica plating of some spore clones on the media indicated (on the left). YPG, Yeast extract-Peptone-Glycerol medium.

All T. delbrueckii strains showed homozygosity for most phenotypes analyzed (fermentation of sucrose, maltose, galactose, melibiose, melezitose, trehalose, raffinose, starch, and $\alpha$-methyl glucoside; copper resistance; $\mathrm{H}_{2} \mathrm{~S}$ production; homothallism; and petite phenotype) except for resistance to $125 \mathrm{mg} / \mathrm{L} \mathrm{SO}_{2}$, for which differences between the spore clones of some tetrads were sometimes observed. Nonetheless, no segregation pattern was observed for any of the phenotypes analyzed. T. delbrueckii strains and their spore clones were resistant to $5 \%$ ethanol but not $10 \%$. Td EX1257, $T d$ EX1257-CYH5, and their spore clones did not resist $125 \mathrm{mg} / \mathrm{L} \mathrm{SO}_{2}$. Td EX1180, Td EX1180-11C4, and their spore clones were resistant to $125 \mathrm{mg} / \mathrm{L} \mathrm{SO}_{2}$ but not to $250 \mathrm{mg} / \mathrm{L}$. All T. delbrueckii strains and their spore clones produced more $\mathrm{H}_{2} \mathrm{~S}$ (brown color on Biggy agar) than S. cerevisiae (beige to light brown). $T d$ EX1257, Td EX1257-CYH5, and their spore clones were resistant to $36 \mathrm{mg} / \mathrm{L}$ copper but not $T d$ EX1180, Td EX1180-11C4, or their spore clones (Figure 1B). This high degree of homozygosity for the phenotypes analyzed makes us suspect that our T. delbrueckii strains are haploid yeasts that mate among themselves or with their daughter buds to originate diploid cells immediately before undergoing meiosis to generate tetrads. Indeed, conjugative tubes were observed in all these yeasts before obtaining spores in SPO medium. This circumstance rules out any possibility of detecting heterozygous loci, unless two strains with different alleles for the analyzed genes mated immediately before sporulation.

\subsection{Fermentation Capability of T. delbrueckii Spore Clones}

To avoid any uncertainty and totally rule out the possible influence of any deleterious allele related to the growth of $T$. delbrueckii that we had not detected, eight large colony-size spore clones from two tetrads of $T d$ EX1180-11C4 (5A, 5B, 5C, and 5D; and 6A, 6B, 6C, and 6D) and another four spore clones from a $T d$ EX1180 tetrad (5A, 5B, 5C, and 5D) were chosen to perform fermentation of synthetic must. All spore clones had slower fermentation kinetics than S. cerevisiae, and were similar to the corresponding parental strain (see Figure 2A for those from Td EX1180-11C4). The increase in cell death during the fermentation of $T$. delbrueckii spore clones was still greater than that of $S$. cerevisiae in all cases, and not very different from that of the parental T. delbrueckii strain (Figure 2B). However, to ensure that 
the new improved yeasts did not contain any deleterious alleles related to growth, the new spontaneous mutants of $T$. delbrueckii were subsequently isolated from some of these well-growing spore clones (see below). This may be important because the presence of any undetected deleterious allele in the parental yeast could cause some fermentation problems in environmental conditions different from those in the present study.
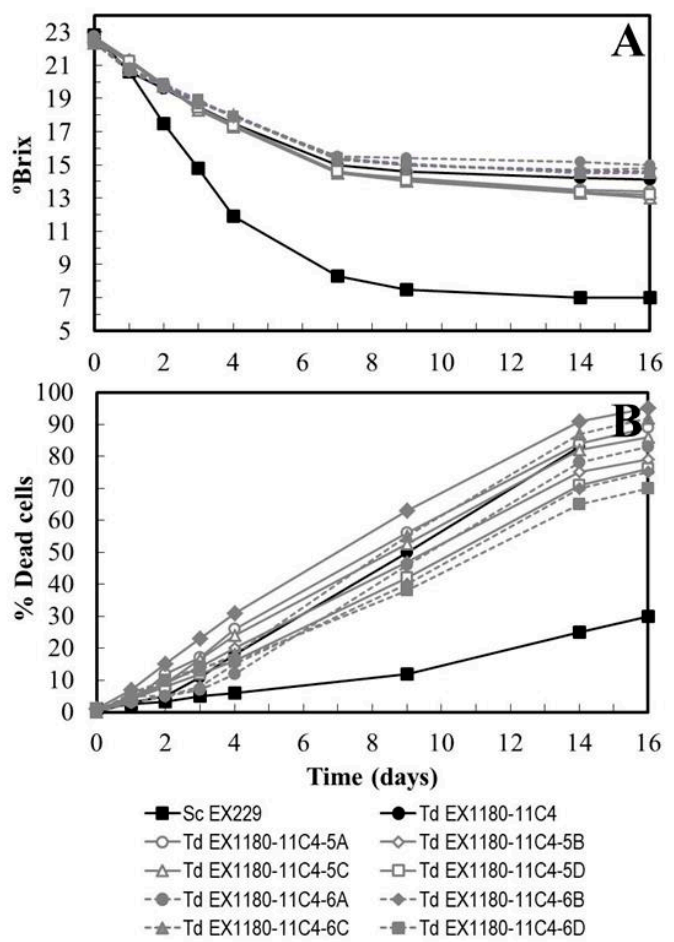

Figure 2. Synthetic must fermentation kinetics of eight large colony size spore clones from two complete tetrads of Td EX1180-11C4 (5A, 5B, 5C, and 5D; and 6A, 6B, 6C, and 6D). (A) Degrees Brix ( ${ }^{\circ}$ Brix) of must/wine. (B) Percentage of dead cells. Data are the mean values of three fermentations inoculated with each yeast strain. Standard deviations were less than $8 \%$ of the means. The degree of dominance throughout fermentation of each inoculated yeast strain was $100 \%$.

\subsection{Isolation and Characterization of New T. delbrueckii Mutants Resistant to $\mathrm{SO}_{2}$ and Ethanol}

Several Td EX1180-11C4 (27) and Td EX1257-CYH5 (18) spore clones were plated onto YEPD plates supplemented with $250 \mathrm{mg} / \mathrm{L} \mathrm{SO}_{2}$. Resistant papillae were isolated only from the Td EX1180-11C4-5B and -6A spore clones. No papilla were isolated from the Td EX1257-CYH5 spore clones. A purified colony was selected from Td EX1180-11C4-5B and -6A papillae: Td Mut5B-SO2R and Td Mut6A-SO2R, respectively. Subsequently, we were able to isolate new spontaneous mutants capable of growing on YEPD plates with $10 \%$ ethanol, but only from Mut6A-SO2R. The authenticity of these new $T$. delbrueckii mutants was verified by analyzing the morphology of their vegetative cells, spores, killer phenotype, cycloheximide resistance, viral dsRNA profile, and mtDNA Restriction Fragment Length Polymorphism (RFLP) profile. The results of all tests agreed with those corresponding to the parental strain Td EX1180-11C4 for all the mutants (i.e., one can rule out that these mutants might have come from other contaminating yeasts). The fermentation capability in synthetic must of the $\mathrm{SO}_{2}$ resistant mutants Td Mut5B-SO2R and Td Mut6A-SO2R was similar to that of their parental yeast Td EX1180-11C4. However, a slight improvement was seen in the $\mathrm{SO}_{2}+$ ethanol resistant mutants (named Td Mut6A-SO2R-EtOHR-31 and Td Mut6A-SO2R-EtOHR-33), although this improvement became irrelevant after 14 days of fermentation (Figure 3A). The fermentation capability of $\mathrm{Td}$ Mut5B-SO2R and Mut6A-SO2R in synthetic must supplemented with $50 \mathrm{mg} / \mathrm{L} \mathrm{SO}_{2}$ was also slightly better than that of the parental strain during the first days of fermentation, but this improvement also 
became irrelevant after the sixth day of fermentation, when approximately $5 \%$ ethanol was reached. However, an evident and relevant improvement was observed in Td Mut6A-SO2R-EtOHR-31 and Td Mut6A-SO2R-EtOHR-33 that was maintained throughout fermentation (Figure 3B). These results indicate that in must fermentation, where there is a rapid increase in ethanol concentration, the possible fermentative improvement that the resistance to $\mathrm{SO}_{2}$ phenotype would provide to T. delbrueckii is only relevant if the yeast strain also has increased ethanol resistance.
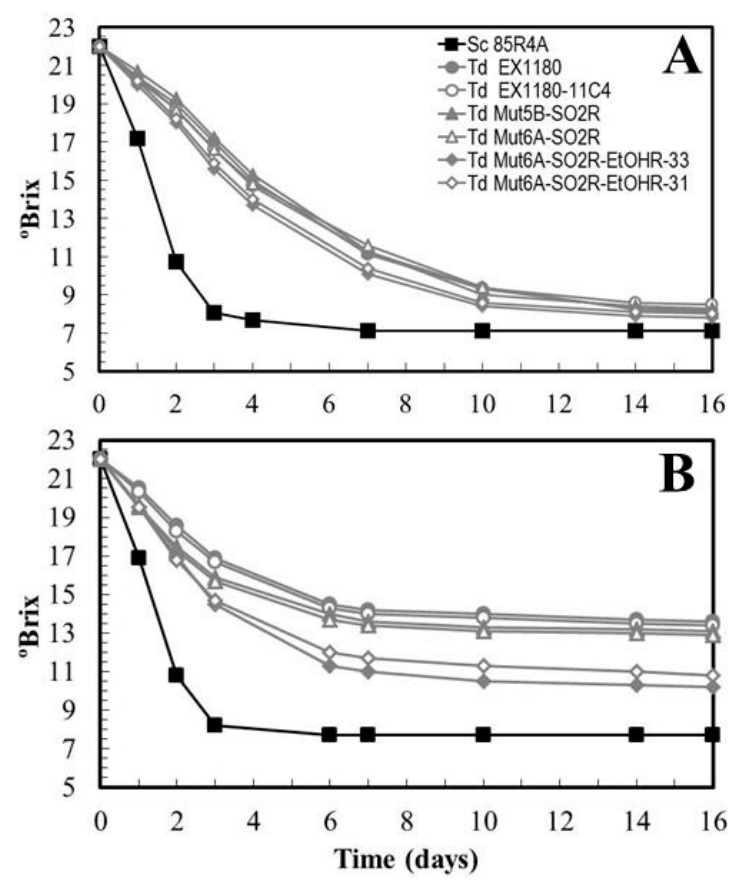

Figure 3. Fermentation kinetics of $T$. delbrueckii $\mathrm{SO}_{2}$ resistant mutants (Td Mut5B-SO2R and Td Mut6A-SO2R) and $\mathrm{SO}_{2}+$ ethanol resistant mutants (Td Mut6A-SO2R-EtOHR-31 and $\mathrm{Td}$ Mut6A-SO2R-EtOHR-33) in synthetic must (A) and synthetic must containing $50 \mathrm{mg} / \mathrm{L} \mathrm{SO}_{2}$ (B). Data are the mean values of three fermentations inoculated with each yeast strain. Standard deviations were less than $10 \%$ of the means. The degree of dominance throughout fermentation of each inoculated yeast strain was $100 \%$.

\subsection{Isolation and Fermentation Capability of New T. delbrueckii Mutants Resistant to High $\mathrm{CO}_{2}$ Pressure} $\left(\mathrm{HP}^{R}\right)$ from Mutants already Resistant to $\mathrm{SO}_{2}$ and Ethanol

One mutant of each type was selected to make rosé sparkling wine (cava) under cellar conditions: Td Mut5B-SO2R (resistant to $\mathrm{SO}_{2}$ ) and Td Mut6A-SO2R-EtOHR-33 (resistant to $\mathrm{SO}_{2}$ and ethanol). As was the case with the parental strain Td EX1180-11C4, no T. delbrueckii mutant was able to dominate the entire process to the end and complete the second in-bottle fermentation, while this was accomplished successfully by the reference yeast Sc EX229. Fermentation was also successfully accomplished by a mixed inoculum of Sc EX229 + Td EX1180-11C4, but in this case, the T. delbrueckii yeasts were quickly overcome by $S$. cerevisiae yeasts from the beginning of fermentation. All single-inoculated T. delbrueckii yeasts began to die and were overcome by contaminant Saccharomyces yeasts after 20 days of fermentation, when a $\mathrm{CO}_{2}$ pressure of about $1 \mathrm{~atm}$ was reached. Contaminant yeasts are common in industrial base wine, and here were responsible for the completion of fermentations that had been single inoculated with T. delbrueckii. However, in terms of fermentation kinetics and yeast survival together, Td Mut6A-SO2R-EtOHR-33 was better than the parental strain and Td Mut5B-SO2R during the first 40 days of fermentation (Figure 4A,B). The sparkling wines inoculated with the mutants were slightly fruitier, had less aging notes, and greater amounts of some ethyl esters than those inoculated with their parental yeast. However, the relevance of these results is far from clear since how much of this effect was due to the involvement of contaminant Saccharomyces yeasts cannot be specified. 

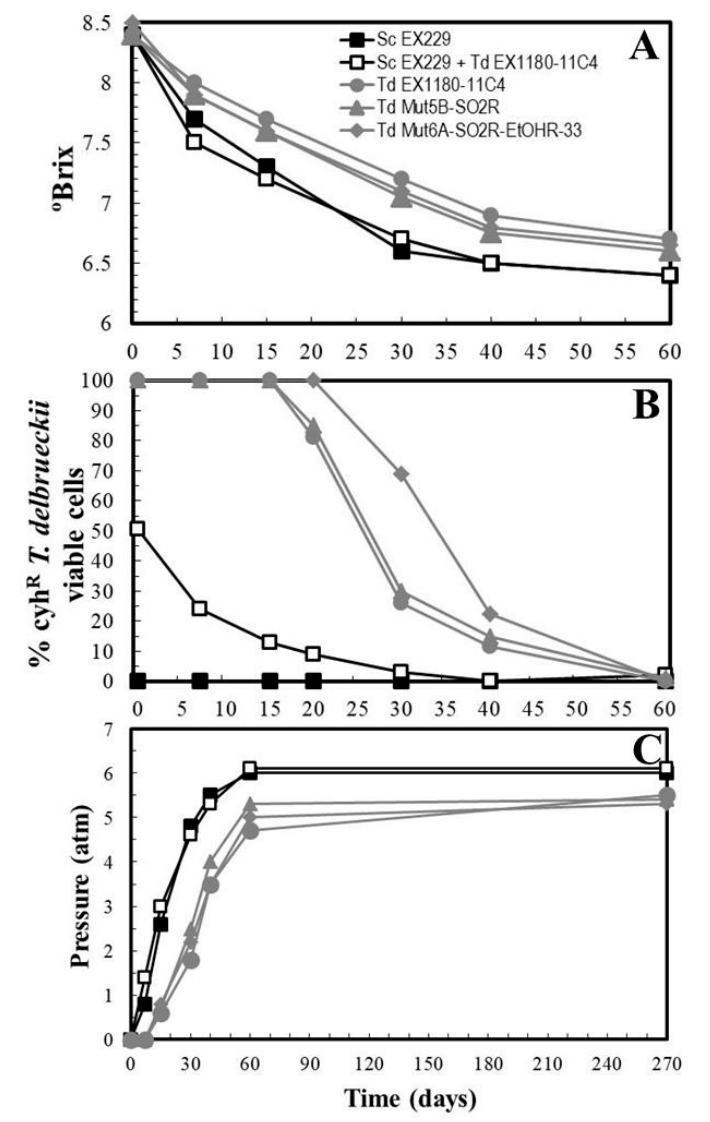

Figure 4. Fermentation kinetics and yeast-population dynamics during sparkling wine second-fermentations inoculated with T. delbrueckii mutants resistant to $\mathrm{SO}_{2}$ and ethanol. Garnacha base wine was single inoculated with Sc EX229, Td EX1180-11C4, Td Mut5B-SO2R or Td Mut6A-SO2R-EtOHR-33, or mixed inoculated with Sc EX229 + Td EX1180-11C4. (A) Evolution of sugar consumption ( ${ }^{\circ}$ Brix). (B) Percentage of $\mathrm{cyh}^{\mathrm{R}}$ yeast cells in each fermentation. Note that the $\mathrm{cyh}^{\mathrm{R}}$ T. delbrueckii viable cells tended to disappear as $\mathrm{CO}_{2}$ pressure increased. (C) Pressure inside the bottle. Data are the mean values of three fermentations inoculated with each yeast strain. Standard deviations were less than $13 \%$ of the means.

Yeast colonies were isolated on YEPD agar inoculated with samples from the sparkling wines that were single inoculated with T. delbrueckii yeasts taken at 30, 40, and 60 days of fermentation. Those colonies that morphologically seemed to correspond to this species were pre-selected, and those that seemed to be $S$. cerevisiae were discarded. After 60 days, when $4.5 \mathrm{~atm}$ pressure had been surpassed (Figure 4C), no viable T. delbrueckii yeasts were isolated. A total of 40 T. delbrueckii colonies were pre-screened to try to obtain possible spontaneous mutants resistant to high $\mathrm{CO}_{2}$ pressure $\left(\mathrm{HP}^{\mathrm{R}}\right)$ that had survived as long as possible during the second in-bottle fermentation (40 days of fermentation). The identity of these possible $\mathrm{HP}^{\mathrm{R}}$ mutants was verified by analysis of cell morphology, killer phenotype, resistance to cycloheximide, presence of viral dsRNA, RFLPs of mtDNA, and sequencing of Internal Transcribed Spacer of ribosomal DNA (ITS). Subsequently, this pre-selection was restricted to eighteen $\mathrm{HP}^{\mathrm{R}}$ mutants: ten from the sparkling wine inoculated with the parental strain $T d$ EX1180-11C4, two from that inoculated with Td Mut5B-SO2R, and six from that inoculated with Td Mut6A-SO2R-EtOHR-33, with full certainty that these mutants came from the corresponding parental T. delbrueckii and not from any other yeast contaminant.

These eighteen $\mathrm{HP}^{\mathrm{R}}$ mutants were inoculated into Macabeo grape must, synthetic must, and synthetic must with $100 \mathrm{mg} / \mathrm{L} \mathrm{SO}_{2}$. Fermentative vigor and the ability to complete fermentation were analyzed. Some improvement was observed for some HPR mutants (such as Td MutHP41 and $T d$ MutHP42) with respect to their parents in fresh grape must fermentations (Figure 5A), but this 
improvement was less clear in synthetic must (Figure 5B). Fermentation kinetics were in most cases faster in fresh grape must than in synthetic must, and were even slower when $100 \mathrm{mg} / \mathrm{L} \mathrm{SO} 2 \mathrm{had}$ been added. This was especially evident for the Td EX1180-11C4 yeast, which is the most sensitive to $\mathrm{SO}_{2}$ and ethanol. The $\mathrm{HP}^{\mathrm{R}}$ mutants had faster fermentation kinetics than their parental yeast Td Mut6A-SO2R-EtOHR-33, being less affected by the presence of $\mathrm{SO}_{2}$. Unfortunately, no mutant completed this type of fermentation within 16 days, which is more than twice the time (seven days) required by the $S c$ 85R4A reference yeast to complete fermentation (Figure 5C). All these results indicate that the synthetic must fermentation conditions were so severe that $\mathrm{SO}_{2}$ resistance of the mutants was not a definitive advantage for the completion of fermentation. Once again, it seems that the high and rapid increase of ethanol concentration in this type of fermentation was the limiting factor for maintaining the viability and fermentation rate of $T$. delbrueckii yeasts, even though the new mutants were resistant to $\mathrm{SO}_{2}$ and this compound was present, or even that they were more resistant to ethanol and $\mathrm{CO}_{2}$ pressure than their parental yeast.

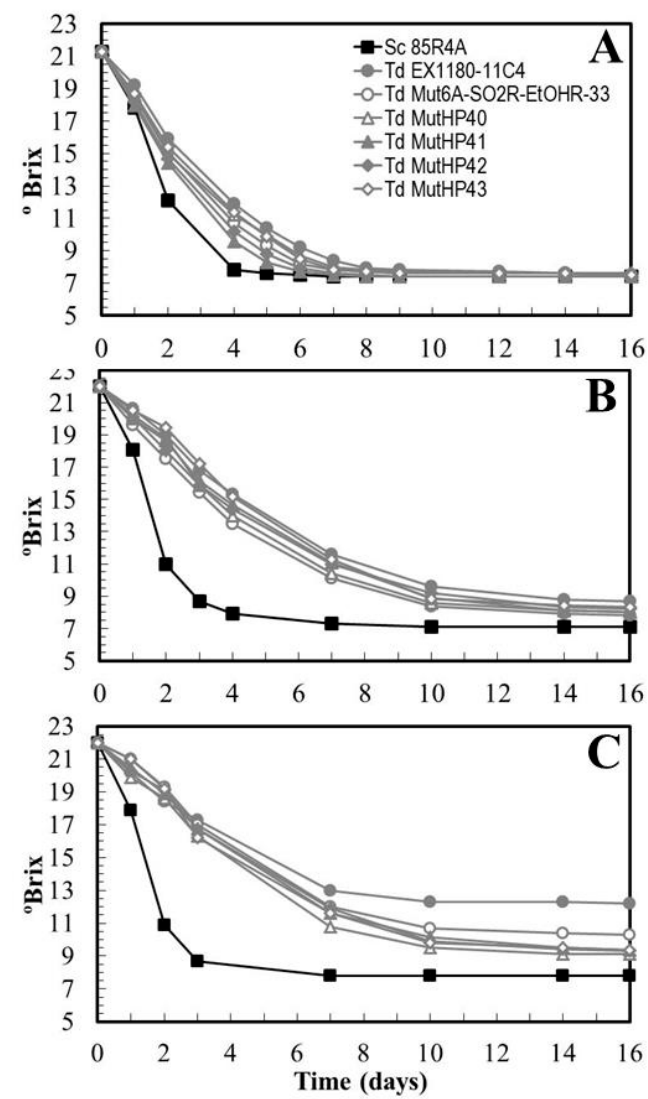

Figure 5. Fermentation kinetics of some T. delbrueckii high $\mathrm{CO}_{2}$ pressure resistant $\left(\mathrm{HP}^{\mathrm{R}}\right)$ mutants inoculated in sterile fresh grape must (A), synthetic must (B), and synthetic must supplemented with $100 \mathrm{mg} / \mathrm{L} \mathrm{SO}_{2}$ (C). Data are the mean values of three fermentations inoculated with each yeast strain. Standard deviations were less than $11 \%$ of the means. The degree of dominance throughout fermentation of each inoculated yeast strain was $100 \%$.

However, most $\mathrm{HP}^{\mathrm{R}}$ mutants showed a relevant improvement in synthetic base wine fermentations supplemented with $50 \mathrm{mg} / \mathrm{L} \mathrm{SO}_{2}$ (Figure 6A). Furthermore, they were able to complete the fermentation when the amount of $\mathrm{SO}_{2}$ was reduced to $30 \mathrm{mg} / \mathrm{L}$ just 3-4 days after the reference yeast Sc 85R4A. Only one of the selected mutants, MutHP40, did not improve with respect to its direct parental strain Td Mut6A-SO2R-EtOHR-33 (Figure 6B). This $\mathrm{SO}_{2}$ concentration is similar to that commonly used in the cava-type sparkling-wine industry (between 15 and $25 \mathrm{mg} / \mathrm{L}$ ). Therefore, the two mutants with the best fermentation kinetics, Td MutHP41 and Td MutHP42, were selected because, in the 
presence of $30-50 \mathrm{mg} / \mathrm{L}$ of $\mathrm{SO}_{2}$, they showed great improvements over their original parental yeast (Td EX1180-11C4) and the intermediate mutant (Td Mut6A-SO2R-EtOHR-33) from which they directly proceeded, and also because they were the mutants whose fermentation kinetics were closest to that of the reference yeast $S c$ 85R4A. Considering the number (18) of initially pre-selected $H \mathrm{P}^{\mathrm{R}}$ clones, one can estimate an $11 \%$ success rate in our strategy to obtain new improved strains of T. delbrueckii for base wine fermentation. However, only some $H P^{R}$ mutants improved the capability for synthetic must fermentation compared to their parental yeast, and this improvement was of little relevance because they did not complete the fermentation in a time that is reasonable for the commercial production of still wines. As noted above, this may be because the increase of ethanol concentration during must fermentation is much greater and faster than in sparkling wine fermentations. In other words, a different, more specific strategy from that used in this present work is probably required to improve the efficiency of $T$. delbrueckii yeasts for still wine fermentation. It was not enough to select mutants resistant to $\mathrm{SO}_{2}$ and ethanol in supplemented culture plates, or $\mathrm{HP}^{\mathrm{R}}$ mutants capable of better resisting the $\mathrm{CO}_{2}$ pressure during the slow second fermentation of traditional sparkling wine.
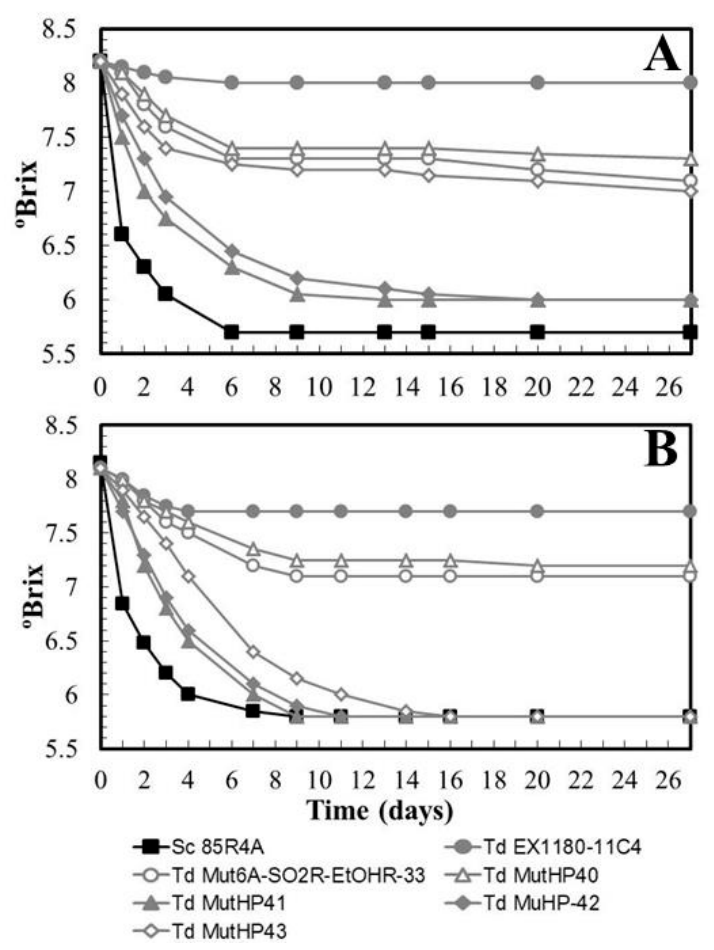

Figure 6. Fermentation kinetics of some T. delbrueckii $\mathrm{HP}^{\mathrm{R}}$ mutants inoculated in synthetic base wine supplemented with $50 \mathrm{mg} / \mathrm{L}$ (A) or $30 \mathrm{mg} / \mathrm{L} \mathrm{SO}_{2}$ (B). Data are the mean values of three fermentations inoculated with each yeast strain. Standard deviations were less than $8 \%$ of the means. The degree of dominance throughout fermentation of each inoculated yeast strain was $100 \%$.

New sparkling wines were made with the two $\mathrm{HP}^{\mathrm{R}}$ selected mutants, $T d$ MutHP41 and Td MutHP42, to repeat the previous strategy for selecting new yeast clones that were even more resistant to high $\mathrm{CO}_{2}$ pressure. Once again, neither of the two inoculated mutants was able to dominate and complete fermentation inside the glass bottle. After day 30, when the pressure reached was greater than $4 \mathrm{~atm}$, the T. delbrueckii yeasts were overwhelmed by the Saccharomyces yeasts contaminating the industrial base wine. This time however, the two mutants had better fermentation kinetics and dominance than their parental yeast. Td MutHP41 was clearly the best of the T. delbrueckii yeasts (Figure 7A-C). These results confirm that $\mathrm{Td}$ MutHP41 is really an improvement for industrial base wine fermentation over its parental yeast. However, neither of the two selected mutants achieved the fermentation speed of the $S$. cerevisiae reference yeast under these cellar conditions. That is, although $T d$ MutHP41 might be able to slowly complete second sparkling wine fermentation, it could well be 
overwhelmed by contaminating Saccharomyces yeasts that can complete fermentation faster than this T. delbrueckii mutant. In this situation, one would always have some uncertainty regarding the final organoleptic quality of commercially produced wines. It has to be borne in mind that wine quality should be equal or very similar in all the bottles of sparkling wine made with the same raw material and under the same cellar conditions.

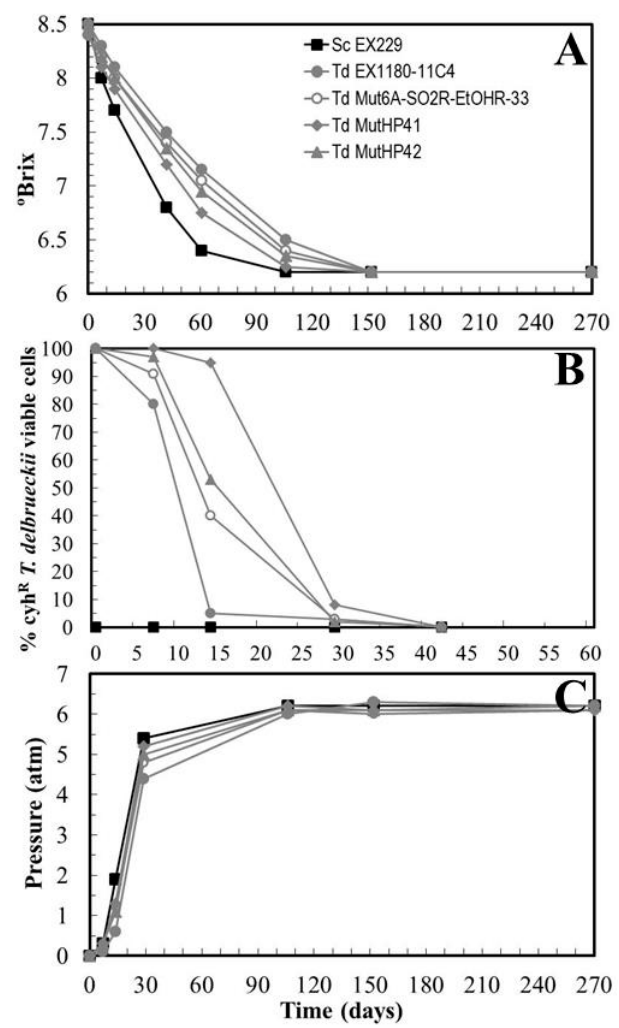

Figure 7. Fermentation kinetics and yeast population dynamics during sparkling wine second-fermentations inoculated with selected T. delbrueckii $\mathrm{HP}^{\mathrm{R}}$ mutants. Macabeo base wine was single inoculated with Sc EX229, Td Mut6A-SO2R-EtOHR-33, Td MutHP41, or Td MutHP42. (A) Evolution of sugar consumption (Degrees Brix). (B) Percentage of $\mathrm{cyh}^{\mathrm{R}}$ yeast cells in each fermentation. (C) Pressure inside the bottle. Data are the mean values of three fermentations inoculated with each yeast strain. Standard deviations were less than $15 \%$ of the means.

We re-isolated new colonies and pre-selected 24 new putative $\mathrm{HP}^{\mathrm{R}}$ clones from the Macabeo sparkling wine samples at the maximum pressure points where viable T. delbrueckii yeasts still remained (14 and 29 days of fermentation). After the appropriate cellular and molecular analyses, 13 clones (five from Td MutHP41 and eight from Td MutHP42) were selected for the fermentation of synthetic wine supplemented with $30 \mathrm{mg} / \mathrm{L} \mathrm{SO}_{2}$. Unfortunately, none of the new preselected clones improved the fermentation kinetics of the parental Td MutHP41 and Td MutHP42. This indicates that repeating the isolation and selection procedure to obtain new reinforced $H P^{R}$ mutants from previously selected $H P^{R}$ mutants was not a sound strategy to continue improving the fermentative capability of T. delbrueckii under high $\mathrm{CO}_{2}$ pressure.

\subsection{Improving the Fermentation Capability of $H P^{R}$ Mutants by Conditioning Yeast Culture before Base Wine Inoculation}

Since T. delbrueckii has a higher oxygen requirement to grow than $S$. cerevisiae, we tested two protocols to condition the yeast cultures prior to inoculation of base wine $\left(30 \mathrm{mg} / \mathrm{L} \mathrm{SO}_{2}\right)$ : one with occasional shaking every $4-12 \mathrm{~h}$ (as is usually done to make commercial sparkling wine), and another with continuous shaking. In this way, one can evaluate how the oxygen supply during the adaptation 
of these yeasts to the base wine environment improves their resistance to ethanol, and hence their fermentation capability. Occasional shaking improved the fermentation capability of all the yeasts, including the $S$. cerevisiae reference strain which already had excellent fermentation capability even without previous culture conditioning. The most relevant improvements were again found for $T d$ MutHP41 and Td MutHP42. Continuous shaking further improved the fermentative capability of all yeasts, and especially that of T. delbrueckii yeasts. Interestingly, Td MutHP42 improved its fermentation capability to values close to $S$. cerevisiae after seven days of fermentation (i.e., once the fermentative capability of the new Td MutHP41 and Td MutHP42 mutants had been improved with respect to the parental T. delbrueckii yeast, it was still possible to further improve their base wine fermentation efficacy by prior conditioning with continuous shaking to provide extra oxygen to the yeast culture) (Figure 8). However, since the fermentation capability of these mutants was still less than that of S. cerevisiae, involvement of this latter yeast may still be necessary to ensure that the second fermentation of sparkling wine is completed in a reasonable time for commercial wineries, preferably within two months. The novelty when using these new $\mathrm{HP}^{\mathrm{R}}$ mutants, conditioned with continuous oxygen supply, is that they would stay alive longer than their parental yeast during second fermentation inside the bottle, thus giving the wine more of the organoleptic characteristics of T. delbrueckii. Furthermore, this is particularly interesting in reducing the inoculum size of these yeasts to the level normally used

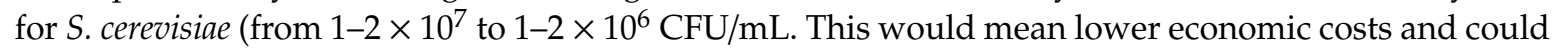
prevent the appearance of odors related to the production of $\mathrm{H}_{2} \mathrm{~S}$ due to the accumulation of excess amounts of dead yeasts.

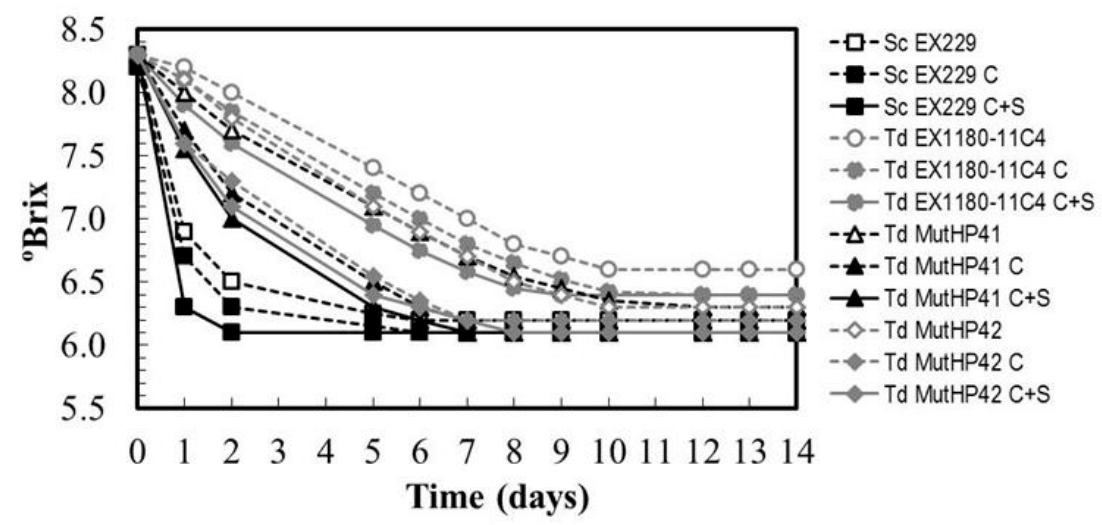

Figure 8. Fermentation kinetics of some T. delbrueckii $\mathrm{HP}^{\mathrm{R}}$ mutants inoculated in synthetic base wine supplemented with $30 \mathrm{mg} / \mathrm{L} \mathrm{SO}_{2}$. Before inoculation, the yeast cultures were unconditioned (no indication following the name of the strain), conditioned with occasional shaking $(\mathrm{C})$, or conditioned with continuous shaking $(\mathrm{C}+\mathrm{S})$. Data are the mean values of three fermentations inoculated with each yeast strain. Standard deviations were less than $9 \%$ of the means. The degree of dominance throughout fermentation of each inoculated yeast strain was 100\%.

\subsection{Genetic Stability of $H P^{R}$ Mutants}

Genetic stability of the new $\mathrm{HP}^{\mathrm{R}}$ mutants after 100 doublings in rich non-selective culture medium (YEPD) was analyzed as previously described [41]. All the mutants maintained the genetic markers after 100 doublings. They also maintained their fermentative capability (Figure 9A,B) and the ability to remain viable longer than their parental strain (Figure $9 C, D$ ) throughout the fermentation of synthetic base wine, regardless of the type of culture conditioning protocol used (Figure 9A,C, vs. Figure 9B,D). This indicates that Td MutHP41 and Td MutHP42 are genetically stable enough to be considered for production at the industrial scale and marketed for use in industrial cellars, with no apparent risk that they might easily lose their new biotechnological properties. We are currently preparing to test these new mutants in commercial cellars for several consecutive years to validate their capability to improve sparkling wine quality, or to make differentiated alternative wines. 


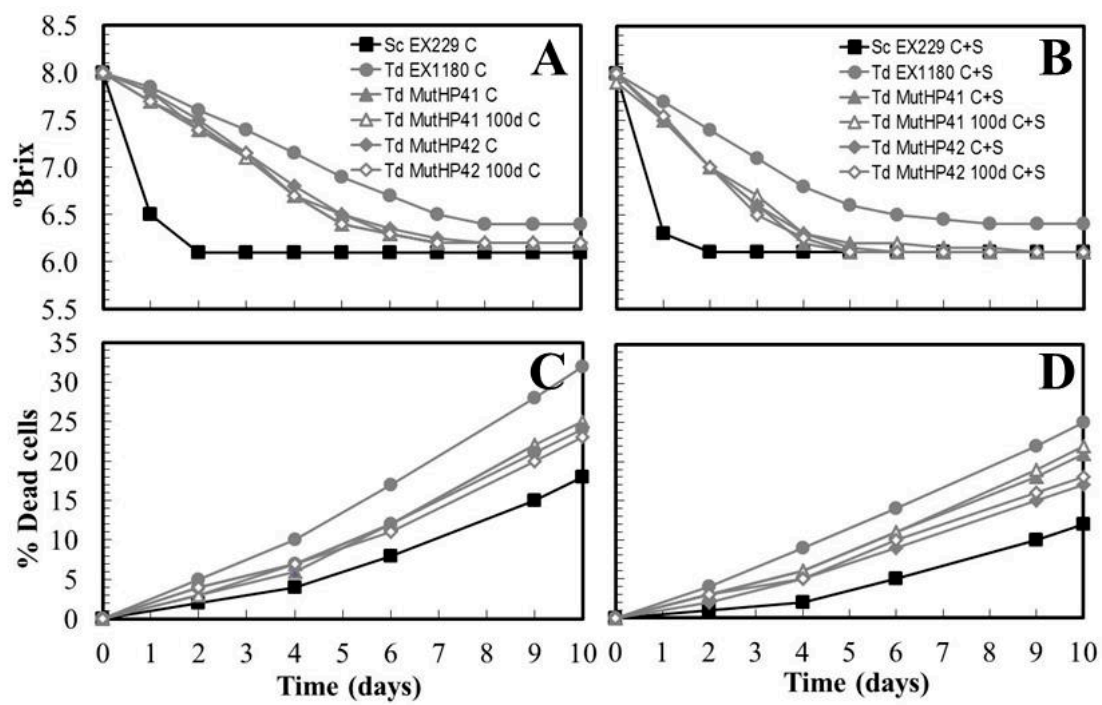

Figure 9. Genetic stability of $\mathrm{HP}^{\mathrm{R}}$ mutants. Fermentation kinetics of synthetic base wine supplemented with $30 \mathrm{mg} / \mathrm{L} \mathrm{SO}_{2}$ and inoculated with the mutants before and after 100 doublings (100d) on YEPD agar. Before inoculation, yeast cultures were conditioned with occasional shaking $(C)$ or continuous shaking $(C+S)$. Data are the mean values of three fermentations inoculated with each yeast strain. Standard deviations were less than $14 \%$ of the means. The degree of dominance throughout fermentation of each inoculated yeast strain was 100\%. (A,C) Yeast cultures conditioned with occasional shaking. (B,D) Yeast cultures conditioned with continuous shaking.

\section{Conclusions}

No deleterious recessive alleles related to cell growth were found in the T. delbrueckii yeasts analyzed, probably because they are haploid strains in their vegetative phase, in which this type of allele would tend to disappear. Consequently, the elimination of alleles of this type cannot be used as a main strategy for the genetic improvement of these non-Saccharomyces yeasts. Isolation of spontaneous mutants resistant to $\mathrm{SO}_{2}$ and ethanol seems to be a good strategy to slightly improve the fermentative efficiency of T. delbrueckii in must and base wine. Sequential isolation of $\mathrm{HP}^{\mathrm{R}}$ mutants from previously obtained mutants resistant to $\mathrm{SO}_{2}$ and ethanol was required to obtain new mutants with significantly improved efficacy for the second fermentation of sparkling wine. These new mutants were genetically stable enough to be considered for industrial production, and their fermentative capability was further improved by continuously supplying oxygen during the conditioning stage before yeast culture inoculation in base wine.

Author Contributions: Conceptualization, M.R.; methodology, M.R., R.V., A.M., E.Z. and M.L.Á.; investigation, M.R., R.V. and A.M.; resources, M.R.; writing_original draft preparation, M.R.; writing-review and editing, M.R., J.B.-G. and L.M.H.; supervision, and; project administration, M.R.; funding acquisition, M.R. All authors have read and agreed to the published version of the manuscript.

Funding: This research was funded by grants GR18117 and IB16132 from the Extremadura Regional Government (Consejería de Economía, Ciencia y Agenda Digital), and AGL2017-87635-R from the Spanish Ministry of Education and Science and the European Regional Development Fund (ERDF, European Union). Rocío Velázquez acknowledges support through a postdoctoral fellowship from the Extremadura Regional Government (file number PO17004, ERDF, European Union).

Conflicts of Interest: The authors declare no conflict of interest. The funders had no role in the design of the study; in the collection, analyses, or interpretation of data; in the writing of the manuscript, or in the decision to publish the results.

\section{References}

1. Benito, S. The impact of Torulaspora delbrueckii yeast in winemaking. Appl. Microbiol. Biotechnol. 2018, 102, 3081-3094. [CrossRef] 
2. Ramírez, M.; Velázquez, R. The yeast Torulaspora delbrueckii: An interesting but difficult-to-use tool for winemaking. Fermentation 2018, 4, 94. [CrossRef]

3. Balmaseda, A.; Bordons, A.; Reguant, C.; Bautista-Gallego, J. Non-Saccharomyces in wine: Effect upon Oenococcus oeni and malolactic fermentation. Front. Microbiol. 2018, 9, 534. [CrossRef]

4. Mauricio, J.C.; Millán, C.; Ortega, J.M. Influence of oxygen on the biosynthesis of cellular fatty acids, sterols and phospholipids during alcoholic fermentation by Saccharomyces cerevisiae and Torulaspora delbrueckii. World J. Microbiol. Biotechnol. 1998, 14, 405-410. [CrossRef]

5. Visser, W.; Scheffers, W.A.; Batenburg-van der Vegte, W.H.; van Dijken, J.P. Oxygen requirements of yeasts. Appl. Environ. Microbiol. 1990, 56, 3785-3792. [CrossRef]

6. Hanl, L.; Sommer, P.; Arneborg, N. The effect of decreasing oxygen feed rates on growth and metabolism of Torulaspora delbrueckii. Appl. Microbiol. Biotechnol. 2005, 67, 11-118. [CrossRef]

7. González-Royo, E.; Pascual, O.; Kontoudakis, N.; Esteruelas, M.; Esteve-Zarzoso, B.; Mas, A.; Canals, J.M.; Zamora, F. Oenological consequences of sequential inoculation with non-Saccharomyces yeasts (Torulaspora delbrueckii or Metschnikowia pulcherrima) and Saccharomyces cerevisiae in base wine for sparkling wine production. Eur. Food Res. Technol. 2015, 240, 999-1012. [CrossRef]

8. García, M.; Greetham, D.; Wimalasena, T.T.; Phister, T.G.; Cabellos, J.M.; Arroyo, T. The phenotypic characterization of yeast strains to stresses inherent to wine fermentation in warm climates. J. Appl. Microbiol. 2016, 121, 215-233. [CrossRef]

9. Velázquez, R.; Zamora, E.; Alvarez, M.L.; Hernández, L.M.; Ramírez, M. Effects of new Torulaspora delbrueckii killer yeasts on the must fermentation kinetics and aroma compounds of white table wine. Front. Microbiol. 2015, 6, e1222. [CrossRef]

10. Ramírez, M.; Velázquez, R.; Maqueda, M.; Zamora, E.; López-Piñeiro, A.; Hernández, L.M. Influence of the dominance of must fermentation by Torulaspora delbrueckii on the malolactic fermentation and organoleptic quality of red table wine. Int. J. Food Microbiol. 2016, 238, 311-319. [CrossRef]

11. García-Ríos, E.; Guillamón, J.M. Sulfur dioxide resistance in Saccharomyces cerevisiae: Beyond SSU1. Microb. Cell 2019, 6, 527-530. [CrossRef]

12. Velázquez, R.; Zamora, E.; Álvarez, M.L.; Ramírez, M. Using Torulaspora delbrueckii killer yeasts in the elaboration of base wine and traditional sparkling wine. Int. J. Food Microbiol. 2019, 289, 134-144. [CrossRef]

13. Caspeta, L.; Chen, Y.; Ghiaci, P.; Feizi, A.; Buskov, S.; Hallström, B.M.; Petranovic, D.; Nielsen, J. Altered sterol composition renders yeast thermotolerant. Science 2014, 346, 75-78. [CrossRef]

14. Lam, F.H.; Ghaderi, A.; Fink, G.R.; Stephanopoulos, G. Engineering alcohol tolerance in yeast. Science 2014, 346, 71-75. [CrossRef]

15. Caspeta, L.; Castillo, T.; Nielsen, J. Modifying yeast tolerance to inhibitory conditions of ethanol production processes. Front. Bioeng. Biotechnol. 2015, 3, 184. [CrossRef]

16. Chen, H.; Jin, S. Effect of ethanol and yeast on cellulase activity and hydrolysis of crystalline cellulose. Enzym. Microb. Technol. 2006, 39, 1430-1432. [CrossRef]

17. Casey, G.P.; Ingledew, W.M.M. Ethanol tolerance in yeasts. CRC Crit. Rev. Microbiol. 1986, 13, $219-280$. [CrossRef]

18. Piper, P.W. The heat shock and ethanol stress responses of yeast exhibit extensive similarity and functional overlap. FEMS Microbiol. Lett. 1995, 134, 121-127. [CrossRef] [PubMed]

19. D'Amore, T.; Panchal, C.J.; Russell, I.; Stewart, G.G. A study of ethanol tolerance in yeast. Crit. Rev. Biotechnol. 1989, 9, 287-304. [CrossRef]

20. Divol, B.; du Toit, M.; Duckitt, E. Surviving in the presence of sulphur dioxide: Strategies developed by wine yeasts. Appl. Microbiol. Biotechnol. 2012, 95, 601-613. [CrossRef]

21. García-Ríos, E.; Nuévalos, M.; Barrio, E.; Puig, S.; Guillamón, J.M. A new chromosomal rearrangement improves the adaptation of wine yeasts to sulfite. Environ. Microbiol. 2019, 21, 1771-1781. [CrossRef] [PubMed]

22. Yuasa, N.; Nakagawa, Y.; Hayakawa, M.; Iimura, Y. Distribution of the sulfite resistance gene SSU1-R and the variation in its promoter region in wine yeasts. J. Biosci. Bioeng. 2004, 98, 394-397. [CrossRef]

23. Zimmer, A.; Durand, C.; Loira, N.; Durrens, P.; Sherman, D.J.; Marullo, P. QTL dissection of Lag phase in wine fermentation reveals a new translocation responsible for Saccharomyces cerevisiae adaptation to sulfite. PLoS ONE 2014, 9, e86298. [CrossRef] [PubMed] 
24. Porras-Agüera, J.A.; Román-Camacho, J.J.; Moreno-García, J.; Mauricio, J.C.; Moreno, J.; García-Martínez, T. Effect of endogenous $\mathrm{CO}_{2}$ overpressure on the yeast "stressome" during the "prise de mousse" of sparkling wine. Food Microbiol. 2020, 89, 103431. [CrossRef] [PubMed]

25. Hagman, A.; Säll, T.; Compagno, C.; Piskur, J. Yeast "make-accumulate-consume" life strategy evolved as a multi-step process that predates the whole genome duplication. PLoS ONE 2013, 8, e68734. [CrossRef] [PubMed]

26. Williams, K.M.; Liu, P.; Fay, J.C. Evolution of ecological dominance of yeast species in high-sugar environments. Evolution 2015, 69, 2079-2093. [CrossRef]

27. Ramírez, M.; Pérez, F.; Regodón, J.A. A simple and reliable method for hybridization of homothallic wine strains of Saccharomyces cerevisiae. Appl. Environ. Microbiol. 1998, 64, 5039-5041. [CrossRef]

28. Ramírez, M.; Regodon, J.A.; Pérez, F.; Rebollo, J.E. Wine yeast fermentation vigor may be improved by elimination of recessive growth-retarding alleles. Biotechnol. Bioeng. 1999, 65, 212-218. [CrossRef]

29. Rodríguez-Cousiño, N.; Maqueda, M.; Ambrona, J.; Zamora, E.; Esteban, E.; Ramírez, M. A new wine Saccharomyces cerevisiae double-stranded RNA virus encoded killer toxin (Klus) with broad antifungal activity is evolutionarily related to a chromosomal host gene. Appl. Environ. Microbiol. 2011, 77, 1822-1832. [CrossRef]

30. Pérez, F.; Regodón, J.A.; Valdés, M.E.; De Miguel, C.; Ramírez, M. Cycloheximide resistance as marker for monitoring yeasts in wine fermentations. Food Microbiol. 2000, 17, 119-128. [CrossRef]

31. Ramírez, M.; Velázquez, R.; Maqueda, M.; López-Piñeiro, A.; Ribas, J.C. A new wine Torulaspora delbrueckii killer strain with broad antifungal activity and its toxin-encoding double-stranded RNA virus. Front. Microbiol. 2015, 6, 983. [CrossRef] [PubMed]

32. Guthrie, C.; Fink, G.R. Guide to yeast genetics and molecular biology. Methods Enzymol. 1991, 194, 3-57.

33. OIV. Guidelines for the Characterization of Wine Yeasts of the Genus Saccharomyces Isolated from Vitivinicultural Environments; Resolutions of the International Organisation of Vine and Wine 2012; OIV-OENO 370-2012; OIV: Paris, France, 2012; pp. 28-29.

34. Henschek, P.A.; Jiranek, V.; Fleet, G.H. Yeast metabolism of nitrogen compounds. In Wine Microbiology and Biotechnology; Harwood Academic Publishers: Chur, Switzerland, 1993; pp. 77-163.

35. Kaiser, C.; Michaelis, S.; Mitchell, A. Methods in Yeast Genetics; Cold Spring Harbor Laboratory Press: New York, NY, USA, 1994.

36. Mortimer, R.K.; Romano, P.; Suzzi, G.; Polsinelli, M. Genome renewal: A new phenomenon revealed from a genetic study of 43 strains of Saccharomyces cerevisiae derived from natural fermentation of grape musts. Yeast 1994, 10, 1543-1552. [CrossRef] [PubMed]

37. Velázquez, R.; Zamora, E.; Álvarez, M.L.; Álvarez, M.L.; Ramírez, M. Using mixed inocula of new killer strains of Saccharomyces cerevisiae to improve the quality of traditional sparkling-wine. Food Microbiol. 2016, 59, 150-160. [CrossRef] [PubMed]

38. Maqueda, M.; Zamora, E.; Rodríguez-Cousiño, N.; Ramírez, M. Wine yeast molecular typing using a simplified method for simultaneously extracting mtDNA, nuclear DNA and virus dsRNA. Food Microbiol. 2010, 27, 205-209. [CrossRef] [PubMed]

39. E.C. $\mathrm{N}^{\mathrm{o}} 761$. Amending Regulation EEC $\mathrm{N}^{\mathrm{o}}$ 2676/90 determining community methods for the analysis of wines. Off. J. Eur. Community 1999, L99, 5-9.

40. Ambrona, J.; Vinagre, A.; Ramírez, M. Rapid asymmetric evolution of Saccharomyces cerevisiae wine yeasts under apparently non-selective conditions. Yeast 2005, 16, 1299-1306. [CrossRef]

41. Ramírez, M.; Vinagre, A.; Ambrona, J.; Molina, F.; Maqueda, M.; Rebollo, J.E. Genetic instability of heterozygous hybrid populations of natural wine yeasts. Appl. Environ. Microbiol. 2004, 70, 4686-4691. [CrossRef]

(C) 2020 by the authors. Licensee MDPI, Basel, Switzerland. This article is an open access article distributed under the terms and conditions of the Creative Commons Attribution (CC BY) license (http://creativecommons.org/licenses/by/4.0/). 